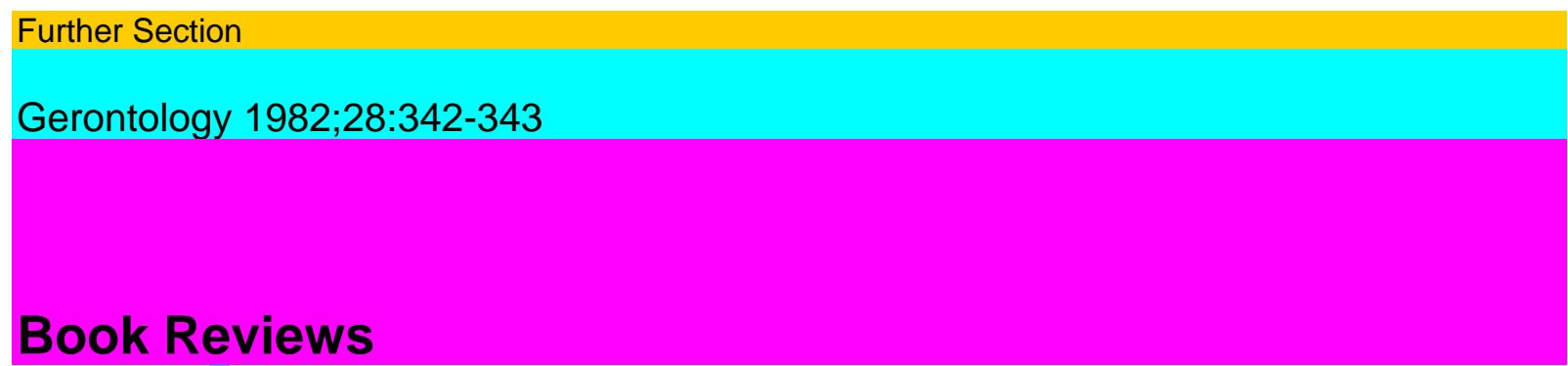

\title{
H.M. Hodkinson
}

An Outline of Geriatrics, 2nd. ed.

Academic Press, London 1981

166 pp.; US \$ 12.-/E4.80

ISBN 0-12-792035-8

This is a useful introduction to the practice of modern Geriatric Medicine and is primarily aimed at Medical Students; junior Doctors contemplating a career in Geriatrics or General Practice, Nursing Staff and Remedial Therapists may well find it useful too. The chapters are written with clarity and are easily comprehensible.

\section{A.A. Diet $\sum$}

Aging - Its Chemistry

American Association for Clinical Chemistry, Inc., Winston-Salem 1980 XXV+ 448 pp.; US\$ 31.00 ISBN 0-915274-10-8

This is one of the proceedings of Arnold O. Beck-man Conference on Clinical Chemistry held in Colorado Springs, USA in 1979. The book has five chapters: (1) Normal aging and senescence; (2) Metabolic changes in aging; (3) Degenerative changes; (4) Experimental approach to aging research; (5) Clinical chemistry. Thus, it covers a wide spectrum. Each chapter has 3-7 papers, and each paper is followed by References. Each chapter has a very useful discussion section in which, as expected, many new points are brought out that have not been presented by the authors. A comprehensive Index is given at the end.

Some of the chapters have outstanding papers, particularly those of Shock, Gordan and Vaughan, Hayflick, Hart and Stephens, and both the papers of Grannis. In Chapter 1, Shock gives an excellent and up-to-date overview of the physiological aspects of aging, a study in which he has been involved for decades. He brings out ably the distinction between chronological age and physiological age, and understandably concludes that there is no general 'age' factor. He suggests ways to choose persons for different

types of work by conducting various tests and having their functional profiles. How far it would be practicable remains to be seen. The paper of Strehler on 'A critique of theories of biological aging' is not a critique. It is superficial. Particularly, his conclusions regarding percentage probable contributions ( $\% \mathrm{PC}$ ) of various factors to aging is incomprehensible. Certainly, aging research has advanced to a level when we can distinguish between primary and secondary causes of aging, and a more critical analysis of the theories that deal with changes in genes can be made. The papers in chapter 2 are rather superficial. In Chapter 3, the paper of Gordan and Vaughan on the interrelationship of calcium and reproductive hormones of both females and males is well brought out. Not much basic research has been done on the contribution of bone to aging, and in the light of the recent finding that calmodulin regulates calcium level it may be worthwhile to pursue this aspect at depth. In chapter 4, Hayflick gives a good and comprehensive account of cellular aging, and brings home the relevance of such work. Supporting data from transplantation studies are given. He touches upon the basic aspects of aging which are missing elsewhere in the 
book. Even though Hart and Stephen have quite well brought out the point that efficient DNA repair mechanism may be responsible for extension of longevity in mammals, whether the various enzymes involved in DNA repair show changes in level and activity in the species are not touched.

The two papers of Grannis in chapter 5 are outstanding. His approach to the problem and analysis are refreshing. He scans the entire life span very logically because the rate of aging after 60 may be affected by what has happened to the body in earlier years. The concept of breakdown of homeostatic machinery is not new, but he considers it from a theoretical angle and illustrates well this point with several examples, and extends it to the level of the gene. Both the papers are thought-provoking and would be useful not only to those interested in clinical aspects of aging, but also to the biologists studying aging. His demise at such an early age is indeed a great loss to the science of aging. The paper of Bender on how drugs are dealt with in the body is informative from a clinical angle.

Book Reviews

343

Even though the title of the book is 'Aging - Its Chemistry' there is not much Chemistry in it. It is rather descriptive. There are about 10 (of a total of 24) good papers which would be of value to all those interested in aging research. It would be useful as a reference book, particularly for physiologists and clinicians interested in aging.

M.S. Kanungo, Varanasi

and psychogeriatricians, particularly those in training, and for doctors who are thinking of embarking on such training. It would also appeal to all those doctors and others, both in the United Kingdom and elsewhere, who wish to understand the nature of current practice in medical and psychiatric departments that care for the elderly.

J. Andrews, Isleworth

Tom Arie

Health Care of the Elderly

Croom Helm, London 1981240 pp.; E 13.95 ISBN 0-7099-0252-2

When inviting contributors to this book, the editor, Prof. Tom Arie, Psychogeriatrician and Professor of Health Care of the Elderly at the University of Nottingham, England, asked for a personal essay rather than a review or a report of work done. The emphasis was to be on ideas and the main concern to catch the reader's interest, in order to produce an 'unstuffy book on very serious matters'. The book contains 14 essays, all but two written by geriatricians or psychogeriatri-cians working in academic centres on both sides of the Atlantic. The exceptions among the authors are a neurologist and a Professor of Social Work.

All of the essays are full of interesting insights and provocative comments. Two maintain the standard set by the editor throughout. Dr. Boyd, a geriatrician from Nottingham, goes to the heart of the nature of geriatric medicine when discussing 'What is a "social problem" in geriatrics'.

Prof. Isaacs, Professor of Geriatric Medicine in Birmingham, contributes a piece full of bravura entitled 'Is geriatrics a specialty?'

The merit of this book is that the whole is greater than the sum of its parts. By considering all of it, the reader will gain a flavour of both the value of working specifically with the elderly for the patients and the community and the fascination which such work provides. It will be valuable reading for all geriatricians 
Edward B. Elkowitz

Geriatric Medicine for the Primary Care

Practitioner

Springer, New York 1981226 pp.; US \$ 21.50 ISBN 0-8261-3230-8

A textbook on Geriatric Medicine for the Primary Care Practitioner should concern itself both with the medical problems common in the elderly, particularly those associated with multiple pathology, and with the social problems connected with retirement and social isolation. This book unfortunately fulfils neither of these aims nor is there a separate chapter on psychiatry. The author subsequently states that his concern is for 'a whole person' medicine, but then goes on to discuss each pathological process, disease by disease and system by system, in isolation. Multiple small inaccuracies also somewhat irritate the reader, such as: (a) 'It (of Hodgkin's disease) occurs in $10 \%$ of the elderly over 60 years of age' - what he presumably means is that $10 \%$ of the cases of Hodgkin's disease occur in the elderly, not that $10 \%$ of the elderly have Hodgkin's disease, (b) 'Post-menopausal bleeding is most commonly caused by vaginitis in the case of spot bleeding' - this seems somewhat unlikely and therefore requires an original reference to validate this statement.

Unfortunately, one cannot recommend this book for those of the primary care team specially concerned with the elderly. 